\title{
DETERMINACIÓN DE CULTIVOS AGRÍCOLAS Y FIRMAS ESPECTRALES MEDIANTE EL PROCESAMIENTO DE IMÁGENES SATELITALES UTILIZANDO EL MÉTODO DE CLASIFICACIÓN SUPERVISADA
}

\author{
DETERMINATION OF AGRICULTURAL CROPS AND SPECTRAL \\ SIGNATURES BY PROCESSING SATELLITE IMAGES USING \\ THE SUPERVISED CLASSIFICATION METHOD
}

\author{
Javier H. Rivas León ${ }^{1}$
}

RECEPCIÓN: 29 DE OCTUBRE DE 2020

ACEPTACIÓN: 18 DE NOVIEMBRE DE 2020

\section{RESUMEN}

La presente investigación corresponde al procesamiento de imágenes de satélite con la finalidad de mostrar los procesos de clasificación y la variabilidad multitemporal basada en la dispersión de píxeles codificados. A partir de la observación de las variaciones en los usos de suelos destinados a la actividad agraria en el valle de Chincha, se muestran incrementos en áreas de cultivos como el algodón (6.55\%) y disminución significativa en otras áreas de cultivo como el de camote (48.09\%). Asimismo, la obtención de firmas espectrales de cultivos como algodón, alcachofa, camote y cítricos, los cuales podrán ser utilizados como fuente de datos para futuros sistemas de monitoreo inteligente con robots y drones aplicados a la agricultura.

Palabras clave: teledetección, firma espectral, clasificación supervisada.

\begin{abstract}
The present research corresponds to the processing of satellite images, in order to show the classification processes and the multitemporal variability based on the dispersion of coded pixels, on which you can observe the variations in the uses of land destined for agricultural activity in the Chincha valley, showing increases in cultivated areas such as cotton (6.55\%) and significant decrease in cultivated areas such as sweet potato $(48.09 \%)$; also obtaining spectral signatures of crops such as; cotton, artichoke, sweet potato and citrus, which can be used as source data for future intelligent monitoring systems with robots and drones applied to agriculture.
\end{abstract}

Keywords: remote sensing, spectral signature, supervised classification.

1 Docente de las asignaturas Mecatrónica aplicada al agro y al gas, Circuitos Electrónicos, Inmótica y Sistemas de Información Geográfica aplicada a la Ingeniería de las Escuelas Profesionales de Ingeniería Mecatrónica y Civil de la Universidad Ricardo Palma. Cuenta con estudios de maestría en Telecomunicaciones, Docencia y Gestión Universitaria; experto en electrónica y geomática. <javier.rivas@urp.edu.pe> 


\section{INTRODUCCIÓN}

La clasificación multiespectral es el proceso de ordenar los datos (píxeles) en un número finito de clases individuales o categorías de datos geoespaciales, los cuales están basados en una categoría de niveles digitales [1]. Si un píxel satisface cierto conjunto de criterios, el píxel es asignado a la clase que le corresponde, llamándosele a este proceso como segmentación de la imagen. Este estudio de investigación se realizó siguiendo las siguientes fases mostradas en la figura 1.

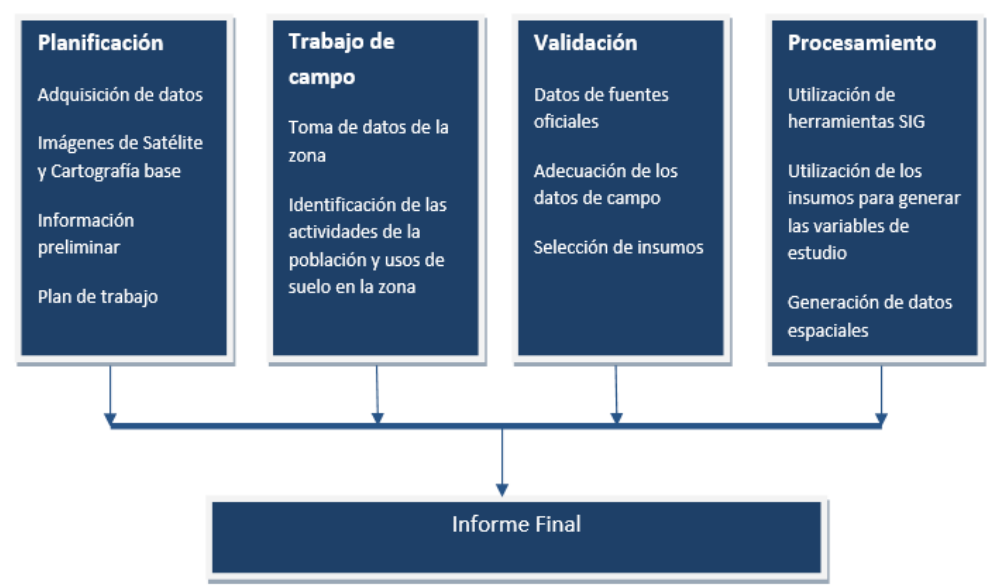

Figura 1. Fases del desarrollo de la investigación. Elaboración propia.

\section{PROCESAMIENTO DE IMÁGENES SATELITALES}

\subsection{Clasificación no supervisada}

Esta etapa corresponde a la adquisición de datos y documentos geoespaciales que sirven de apoyo al proceso de trabajo de campo, para lo cual se realiza un proceso de clasificación no supervisada de imágenes de satélite de la zona de estudio. La finalidad de este procedimiento es obtener datos de referencia sobre los usos de suelos y zonas de cultivo presentes en el área de interés. Esto puede visualizarse en la figura 2.

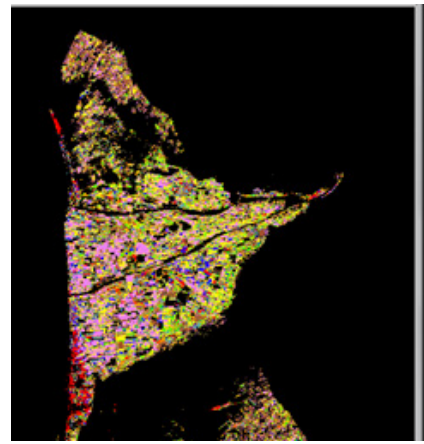

A

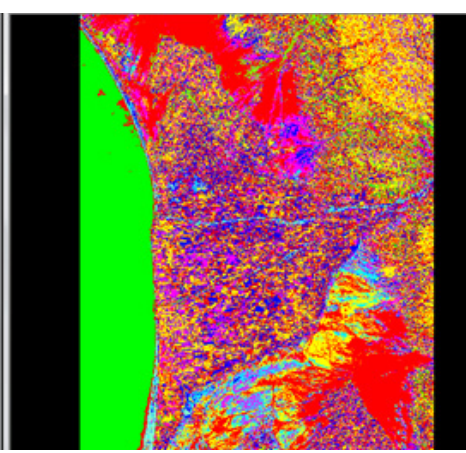

$\mathrm{B}$

Figura 2. Clasificación no supervisada del valle de Chincha. A: Vista general / B: Vista ampliada Elaboración propia con ERDAS IMAGINE. 


\subsection{Procesamiento de la información}

Luego de realizado el trabajo de campo, en el cual se toman datos referidos a las características del área y los tipos de cultivos observados, se procede a la clasificación de uso de suelos. Para tal fin, se utiliza el método de clasificación supervisada utilizando las siguientes entidades: ríos, límite del valle de Chincha y puntos de toma de datos editados en el software ArcGIS y la composición de la imagen satelital (combinación de bandas: 54 2), que utiliza el software Erdas IMAGINE [2] tal como se observa en la figura 3 .
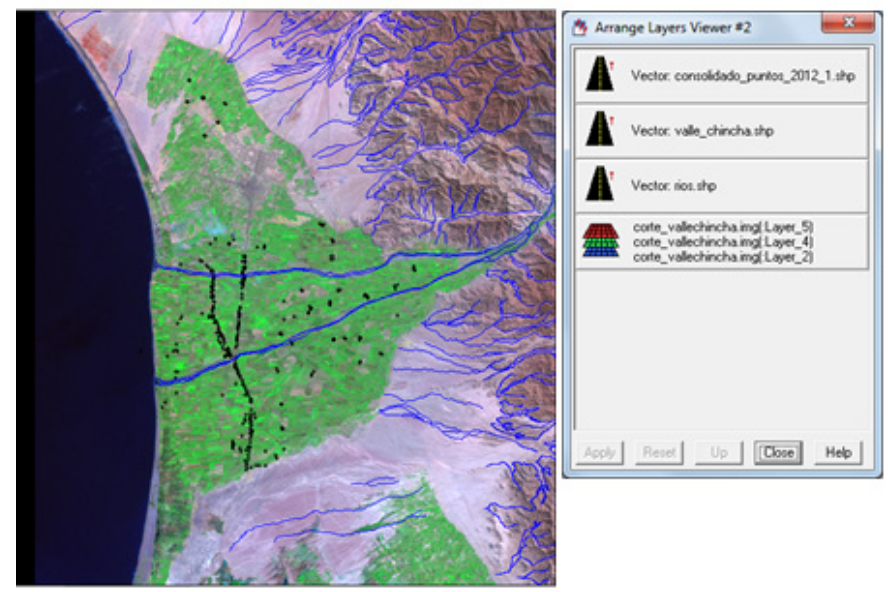

Figura 3. Composición de bandas de la imagen satelital (5 42) y agregación de entidades geoespaciales. Elaboración propia con ERDAS IMAGINE.

Cada punto observado en la figura 3, especifica un tipo de cultivo y un valor de píxel, para los cuales se edita el símbolo con el dato que los representa [2]. Esta dispersión clasifica los tipos de cultivos presentes, entre los cuales se cuenta el algodón, la alcachofa, el camote y los cítricos. A partir de esto, se procesan las curvas de respuesta espectral de los diversos puntos en las zonas cultivadas con el fin de observar cuáles son las tendencias y lograr obtener la firma espectral de cada uno de ellos.

En la figura 4, se visualizan los perfiles espectrales por tipo de cultivo, donde las líneas de color verde siguen una tendencia central respecto del mismo, mientras que las de color rojo no siguen la tendencia, por lo que son descartadas y no son tomadas en cuenta para el proceso de clasificación.

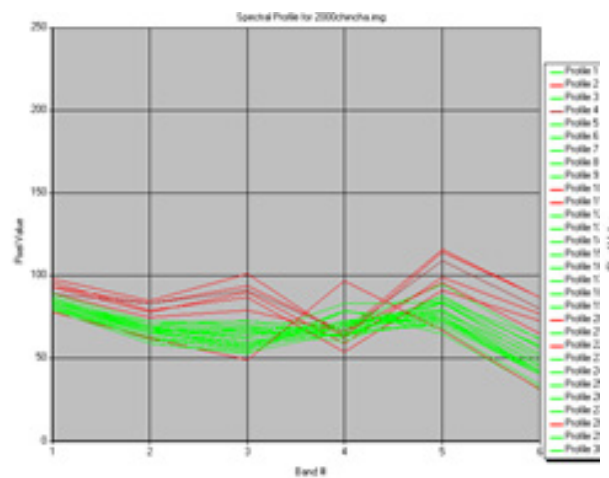

A

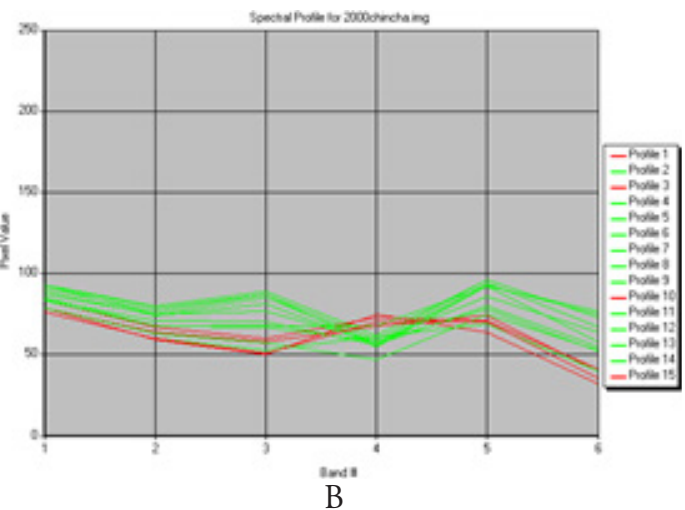

B 


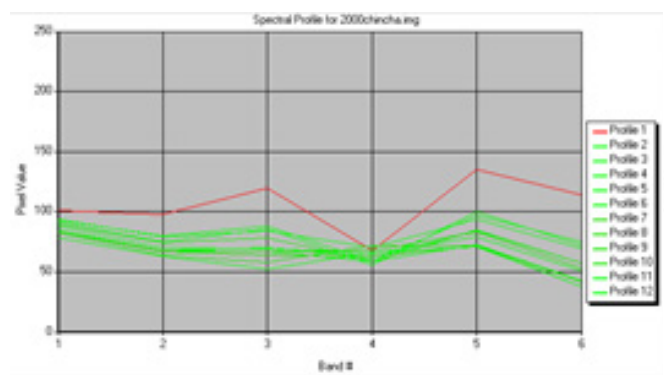

C

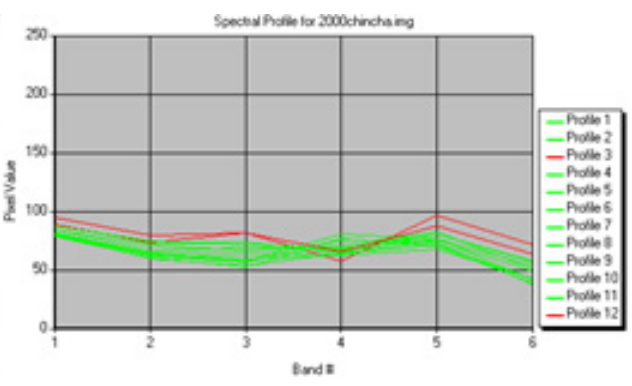

$\mathrm{D}$

Figura 4. Clasificación de respuestas espectrales de los cultivos.

A: algodón / B: alcachofa / C: camote / D: cítricos

Elaboración propia con ERDAS IMAGINE.

A partir de la determinación de cultivos, se procede a realizar una reclasificación (recode) de los atributos con el fin de incluir todos los elementos geoespaciales que integran el estudio y representen la realidad. Para lograrlo, se incluyen los nombres de clases (uso de suelo o tipo de cultivo), codificación de color, histogramas y codificaciones del formato RGB [3], tal como se observa en la figura 5.
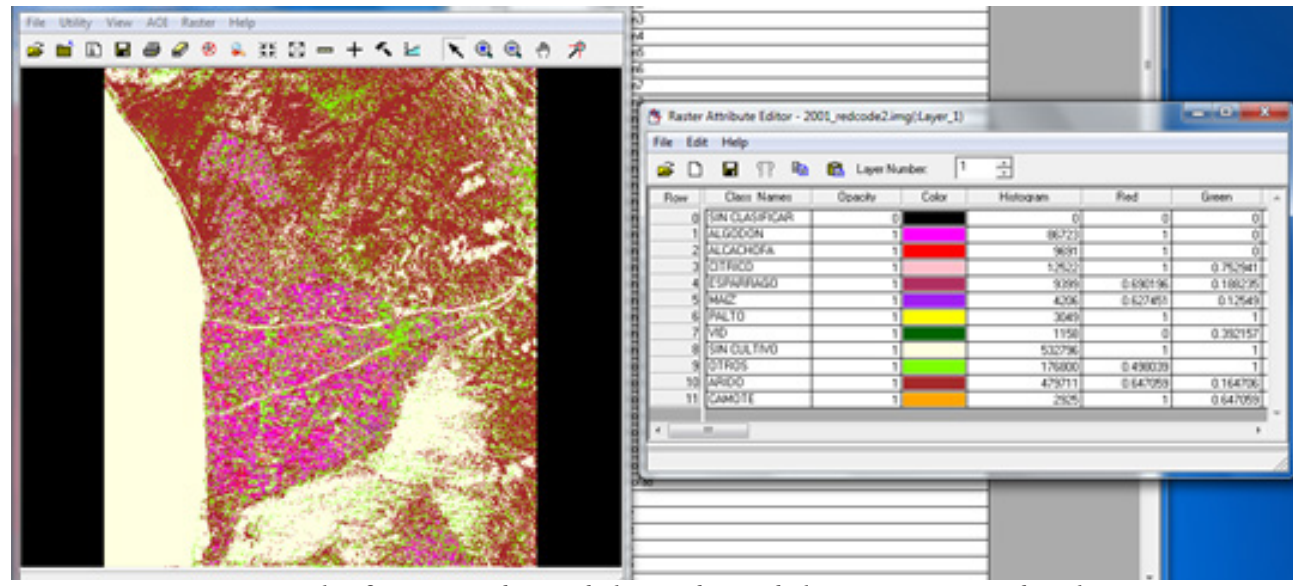

Figura 5. Clasificación y edición de los atributos de las áreas por tipo de cultivo. Elaboración propia con ERDAS IMAGINE.

\subsection{Máscaras y clasificación de atributos}

Realizada la etapa de recodificación de los atributos comprendidos en el área de evaluación, se obtiene la máscara de clasificación multiespectral, en la cual el universo de píxeles es categorizado por tipo de cultivo y definido por su forma de parcela, tal como se muestra en la figura 6 . En ella, las zonas oscuras representan a los cultivos de algodón y cítricos, por tratarse de los mayores tipos de cultivo identificados en el lugar de estudio. 


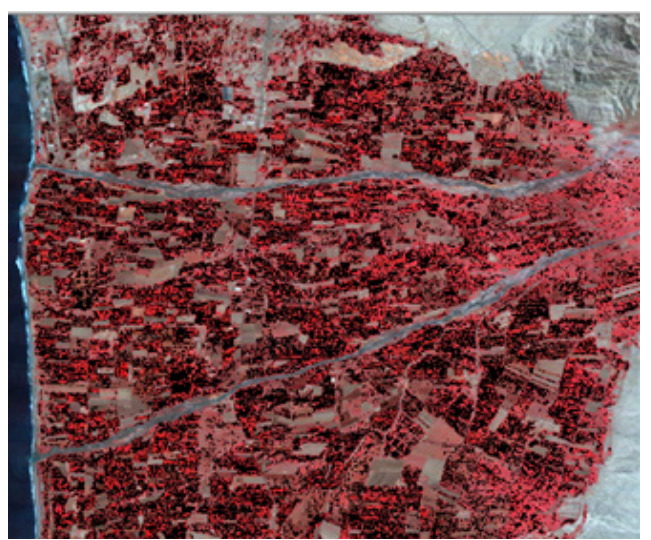

Figura 6. Máscara de clasificación multiespectral de las áreas de cultivo. Elaboración propia con ERDAS IMAGINE.

El proceso de realce de la imagen, clasificada por tipo de cultivo, se realiza mediante el siguiente procedimiento:

Overlay: se realiza un proceso de codificación de la imagen.

Comparación de overlay: para realizar correcciones de valor de píxeles, se sigue una corrección radiométrica del vecino más cercano $3 \times 3$.

La corrección de vecino más cercano: permite que las áreas de los cultivos puedan distinguirse con mayor detalle.

Realizada esta etapa, se observa en la figura 7 el realce de píxeles. Así, se muestra en la tabla de atributos (Histogram, Class Name, Color) la clasificación de usos de suelos por tipo de cultivo, representado en tonalidad de coloración RGB. Se puede identificar en la imagen la dispersión y tipos de cultivos presentes en el área de estudio.

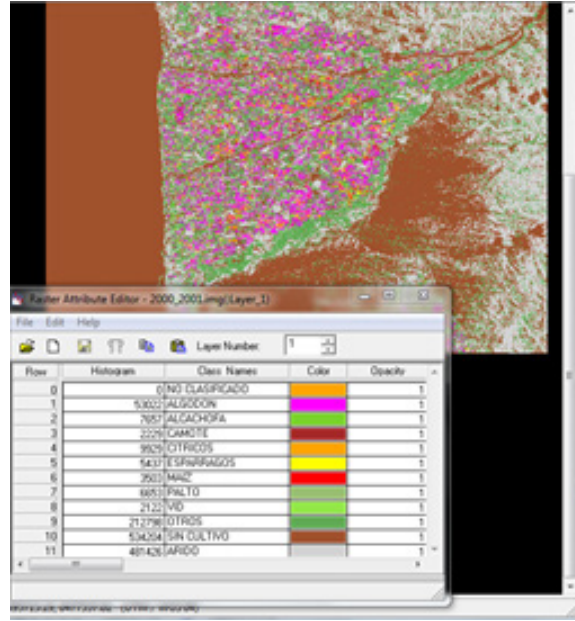

A

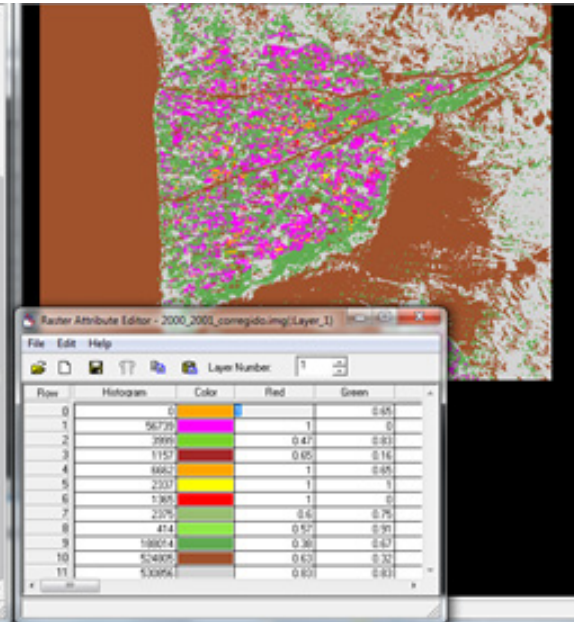

$\mathrm{B}$

Figura 7. Comparación de tablas de atributos de la clasificación multiespectral de las áreas de cultivo. A: Imagen y tabla de atributos sin corrección / B: Imagen y tabla de atributos corregida Elaboración propia con ERDAS IMAGINE. 


\section{RESULTADOS}

\subsection{Comparación multitemporal de variabilidad de uso de suelos}

Los resultados de clasificación multiespectral de las áreas de cultivo son desarrollados, finalmente, en un proceso de unión matricial, en la cual se realiza una operación comparativa multitemporal entre los datos geoespaciales (píxeles) resultantes. La finalidad es analizar la variabilidad de los usos de suelos y/o rotación de cultivos en un periodo dado, para lo cual se deberán ajustar los valores de píxel, de acuerdo con lo mostrado en la figura 8 . En ella, las columnas recode 01 b y recode 3 corresponden a la clasificación supervisada en diferentes periodos de tiempo.

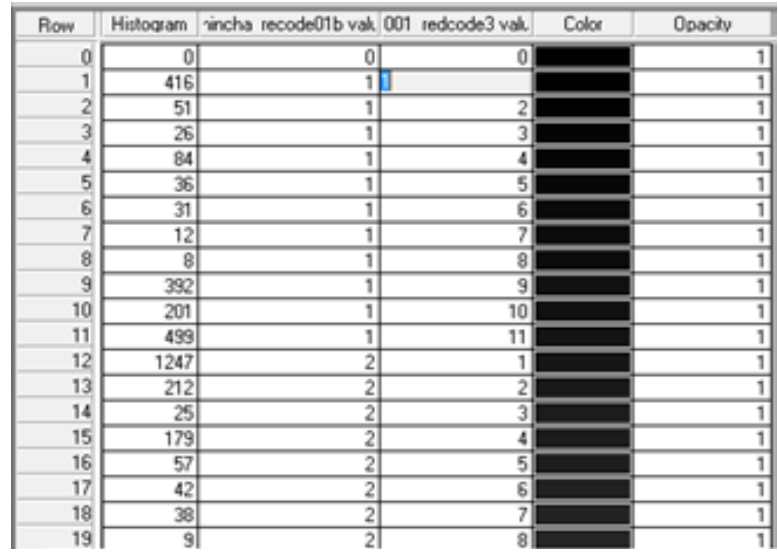

Figura 8. Edición de la unión matricial para la comparación temporal del uso de suelos. Elaboración propia con ERDAS IMAGINE.

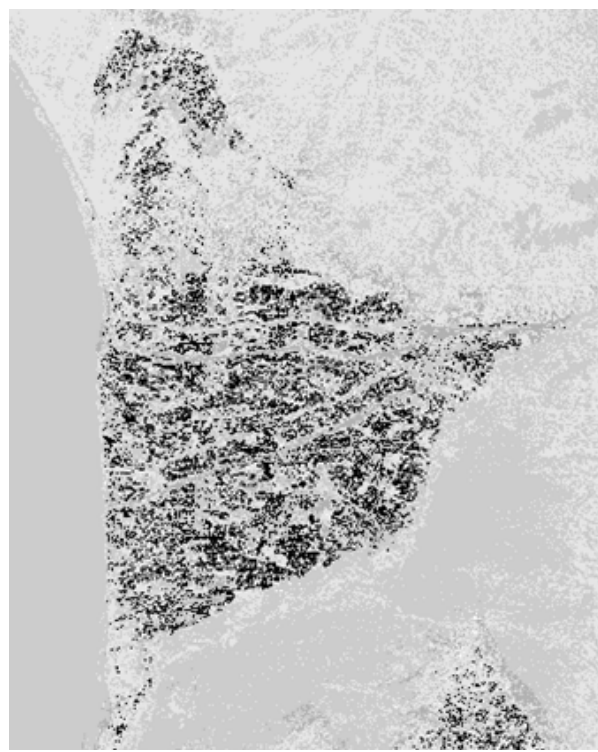

Figura 9. Resultado de la superposición de los datos geoespaciales para la comparación temporal del uso de suelos y variabilidad de cultivos aplicados. Elaboración propia con ERDAS IMAGINE. 
Realizada la etapa de recodificación de los datos de píxel correspondientes a uno de los periodos de tiempo, se aplica la operación de superposición de datos geoespaciales (overlay). De esta manera, se mostrará la variabilidad de usos de suelos sobre los cultivos (ver figura 9) y analizar el grado de productividad que se tienen sobre los mismos en dos periodos de tiempo diferentes [4].

\subsection{Estimación de áreas por valor de píxel}

Corresponde en esta sección realizar la estimación del área total de las zonas cultivables por tipo de cultivo seleccionados para el estudio (algodón, alcachofa, camote y cítricos). Para esto, se cuenta con una resolución espacial de 30m (fuente de imagen satelital: LandSat 7, sensor ETM+) y se aplicará una tabla comparativa con los valores del histograma de clasificación multiespectral [5] mostrados en la figura 7. A y 7. B. Esto se muestra en la tabla 1.

TABLA 1. Cálculo de áreas de uso de suelos por tipo de cultivo siguiendo los patrones de histéresis multitemporales.

\begin{tabular}{|l|r|r|r|r|r|r|}
\hline \multirow{2}{*}{$\begin{array}{c}\text { Nombre } \\
\text { clase de }\end{array}$} & \multicolumn{3}{|c|}{$\begin{array}{c}\text { Imagen } \\
\text { Recode01b }\end{array}$} & \multicolumn{3}{c|}{$\begin{array}{c}\text { Imagen } \\
\text { Recode3 }\end{array}$} \\
\cline { 2 - 7 } & $\begin{array}{c}\text { Histograma } \\
\text { (pixel) }\end{array}$ & $\begin{array}{c}\text { Área } \\
\left(\mathrm{m}^{2}\right)\end{array}$ & $\begin{array}{l}\text { Área } \\
(\mathrm{Ha})\end{array}$ & $\begin{array}{c}\text { Histograma } \\
(\text { pixel })\end{array}$ & $\begin{array}{c}\text { Área } \\
\left(\mathrm{m}^{2}\right)\end{array}$ & $\begin{array}{c}\text { Área } \\
(\mathrm{Ha})\end{array}$ \\
\hline Algodón & 53022 & 1590660 & 159.07 & 56739 & 1702170 & 170.22 \\
\hline Alcachofa & 7657 & 229710 & 22.97 & 3999 & 119970 & 12.00 \\
\hline Camote & 2229 & 66870 & 6.69 & 1157 & 34710 & 3.47 \\
\hline Cítricos & 9929 & 297870 & 29.79 & 6662 & 199860 & 19.99 \\
\hline
\end{tabular}

Elaboración propia.

De la tabla 1, se puede observar, en la comparación multitemporal de los datos geoespaciales procesados, que:

El cultivo de algodón es la principal actividad en la zona de estudio, lo que explica el incremento de $111510 \mathrm{~m}^{2}$ adicionales con respecto a la campaña agrícola anterior. Esto significa un incremento del $6.55 \%$ de áreas cultivadas.

Las áreas correspondientes a los cultivos de alcachofa, camote y cítricos tuvieron una caída de 109 740, 32160 y $98010 \mathrm{~m}^{2}$ respectivamente, lo cual significa una disminución porcentual de $47.77 \%$, $48.09 \%$ y $33.90 \%$ de áreas cultivadas de estas especies.

\section{CONCLUSIONES}

El procesamiento de imágenes satelitales mediante métodos de clasificación supervisada permite generar fuentes de datos referidas a las tendencias de uso de suelos y diversificación de cultivos en un área geográfica específica de evaluación y aplicar la multi-temporalidad (overlay) para observar en una cartografía temática los comportamientos y niveles de producción que se aplican en estas zonas de manera precisa.

De los resultados de la tabla 1 , se observa que la principal actividad agrícola de la zona de estudio es el algodón, pues presentó un incremento de $6.55 \%$ de áreas cultivadas respecto a la campańa anterior. Se concluye que esto es consecuencia de la dinámica que tiene el mercado textil a nivel nacional 
e internacional, lo que permite que una importante proporción de uso de suelos esté destinada a este tipo de cultivos y ha desplazado a otros cultivos como la alcachofa $(-47.77 \%)$ y el camote $(-48.09 \%)$ que han sufrido un significativo descenso de áreas cultivadas.

También se obtuvo la clasificación de las respuestas espectrales de los cultivos presentes en la zona como el algodón, el camote, la alcachofa y los cítricos, los cuales pueden considerarse de suma importancia, ya que estos datos representan la firma espectral de cada especie clasificada. Estos pueden ser utilizados como insumos de datos para futuros procesos inteligentes de monitoreo y supervisión del desarrollo (fenología) y calidad del cultivo, a través de sistemas robotizados y vehículos aéreos no tripulados dispuestos con sensores multiespectrales.

\section{REFERENCIAS}

C. Dallos. "Modelo Para Clasificación de Imágenes Multiespectrales a partir de los Complejos de Células Abstractas (CCA)", tesis de maestría, Universidad Distrital Francisco José de Caldas, 2017 [En línea]. Disponible en: https://repository.udistrital.edu.co/bitstream/handle/11349/5404/ DallosBustosCristianDavid2017.pdf?sequence=1 \&isAllowed=y [Accedido: 28-dic-2020].

E. Posada, H. Ramírez y N. Espejo, Manual de prácticas de percepción remota con el programa ERDAS IMAGINE 2011, Bogotá, Colombia: ONU-IGAC, 2012.

J. Gao, Digital Analysis of Remotely Sensed Imagery, New York, USA: McGraw-Hill, 2009.

C. Pérez y A. Muñoz, Teledetección: Nociones y Aplicaciones, Salamanca, España: Universidad de Salamanca, 2006.

T. Lillesand, R. Kiefer, y J. Chipman, Remote sensing and image interpretation, New York, USA: John Wiley \& Sons, 2008. 